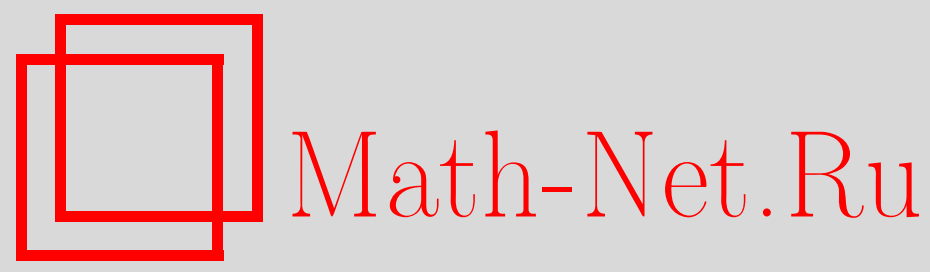

М. Б. Марков, С. В. Паротькин, Моделирование стационарного электромагнитного поля на основе уравнений Максвелла, Матем. моделирование, 2020, том 32, номер 7, 113126

DOI: https://doi.org/10.20948/mm-2020-07-07

Использование Общероссийского математического портала Math-Net.Ru подразумевает, что вы прочитали и согласны с пользовательским соглашением http://www.mathnet.ru/rus/agreement

Параметры загрузки:

IP : 54.80 .73 .141

26 апреля 2023 г., 13:32:51

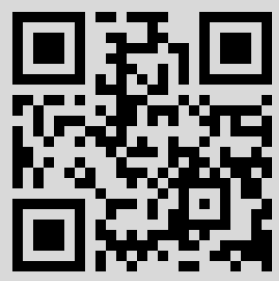




\section{МОДЕЛИРОВАНИЕ СТАЦИОНАРНОГО \\ ЭЛЕКТРОМАГНИТНОГО ПОЛЯ \\ НА ОСНОВЕ УРАВНЕНИЙ МАКСВЕЛЛА}

\section{(C) 2020 2. М.Б. Марков, С.В. Паротькин}

Федеральное государственное учреждение «Федеральный исследовательский центр Институт прикладной математики им. М.В. Келдыша Российской академии наук»

m_b_markov@mail.ru, s_v_p_@mail.ru

\section{DOI: $10.20948 / \mathrm{mm}-2020-07-07$}

Рассмотрена генерация электромагнитного поля в области с идеально проводящей границей импульсом ионизирующего излучения большой длительности. Поставлена задача вычисления поля путем численного решения полной системы уравнений Максвелла. Сформулированы приближения большой и малой радиационной проводимости среды. Для приближений в упрощенных постановках построены аналитические оценки решения уравнений Максвелла. Путем их анализа обоснованы способы вычисления электромагнитного поля в рамках модели, основанной на уравнениях Максвелла в полной постановке. Предложен подход к моделированию поля в постановках, требующих недопустимого объема вычислений для устойчивого решения разностных уравнений Максвелла. Подход позволяет моделировать генерацию электромагнитного поля излучениями космического пространства в аппаратурных блоках с помощью программ, решающих уравнения Максвелла в полной постановке.

Ключевые слова: электромагнитное поле, радиационный пояс, тормозное излучение, уравнения Максвелла, радиационная проводимость, сторонний ток.

\section{MODELING OF THE STATIONARY ELECTROMAGNETIC FIELD BASED ON THE MAXWELL EQUATIONS}

\section{M.B. Markov, S.V. Parot'kin}

Keldysh Institute of Applied Mathematics (Russian Academy of Sciences)

The generation of an electromagnetic field in a region with a perfectly conducting boundary by a long duration pulse of ionizing radiation is considered. The problem of calculating the field by numerically solving the complete system of Maxwell equations is posed. The approximations of the large and small radiation conductivity of the medium in the region are formulated. Analytical estimates of the solution of Maxwell's equations are constructed for approximations in simplified formulations. By analyzing them, methods for calculating the electromagnetic field in the framework of a model based on the Maxwell equations in the full formulation are substantiated. An approach to modeling the 
field in the formulations that require an unacceptable amount of computation for the stable solution of the Maxwell difference equations is proposed. The approach makes it possible to simulate the generation of an electromagnetic field by radiation from outer space in apparatus blocks using programs that solve Maxwell's equations in a complete formulation.

Keywords: electromagnetic field, radiation belt, bremsstrahlung, Maxwell equations, radiation conductivity, external current.

\section{1. Введение}

Взаимодействие излучений космического пространства со спутниками и их носителями сопровождается генерацией помеховых электромагнитных полей. Один из электромагнитных эффектов радиационного происхождения состоит в образовании электромагнитного поля в объемах экранированных блоков аппаратуры. Поле наводит нештатные токи в линиях связи спутника, нарушая функционирование его радиоэлектронной аппаратуры [1].

Математическое моделирование является эффективным методом исследования электромагнитного поля радиационного происхождения. Развитие суперкомпьютерной техники позволяет моделировать электромагнитное поле радиационного происхождения путем непосредственного решения фундаментальных уравнений Максвелла в трехмерной постановке [2].

Полностью консервативная разностная схема для уравнений Максвелла представлена в [3]. Созданы как явный, так и неявный варианты схемы [4]. Авторы данной работы успешно применяли ее для решения практических задач, в которых исследовалось электромагнитное поле, создаваемое импульсными источниками фотонов и электронов [4,5]. В этих задачах произведение длительности воздействующего импульса и скорости света («длина импульса») сравнимо с размером расчетной области.

Моделирование наводок в аппаратуре космических аппаратов, обусловленных воздействием излучений космического пространства, сталкивается с необходимостью вычисления электромагнитного поля на больших временных масштабах. Радиационный пояс Земли создает в аппаратурных блоках космического аппарата электромагнитное поле низкой частоты. Аппроксимация уравнений Максвелла с помощью явной разностной схемы подразумевает выполнение критерия Куранта [6]. Сложная форма космического аппарата требует выбора достаточно малого шага пространственной сетки. Это влечет малый временной шаг. Расчет электромагнитного поля до времен порядка секунды становится нереализуемым даже на суперкомпьютерах. В неявном варианте схема абсолютно устойчива, но соотношение шагов по времени и пространству не может быть слишком велико [7]. 
Данная работа обосновывает способы применения численного алгоритма, основанного на решении уравнений Максвелла в полной постановке, для моделирования электромагнитного поля, создаваемого радиационными воздействиями большой длительности. При этом отработанный численный метод решения уравнений Максвелла явной разностной схемой практически не изменяется. Подход основан на анализе аналитических решений для уравнений Максвелла в упрощенной постановке.

\section{2. Постановка задачи}

Рассмотрим механизм генерации электромагнитного поля в аппаратурном блоке с металлическим корпусом электронами радиационного пояса Земли [8]. Свободные электроны поглощаются в материалах радиационной защиты и в корпусе, генерируя тормозное излучение. Длина пробега фотона тормозного излучения существенно больше, чем у излучившего его электрона [9]. Поэтому фотоны эффективно проникают внутрь аппаратурных блоков. Внутри блок может быть заполнен воздухом или залит диэлектрическим компаундом. В материалах корпусов и в компаунде фотоны испытывают комптоновское и когерентное рассеяния, а также фотопоглощение [10,11]. Фото- и комптоновские электроны отдачи имеют высокую энергию и образуют сторонний электрический ток в корпусе, в компаунде и в воздушной среде. Ударная ионизация материалов электронами отдачи сопровождается образованием в непрерывном спектре вторичных электронов низкой энергии [12-14]. Сторонний ток формирует электромагнитное поле. Электроны низкой энергии дрейфуют в электромагнитном поле, создавая ток радиационной проводимости $[15,16]$. Он компенсирует сторонний ток и снижает электрическое поле. Внутри аппаратурного блока со сплошным металлическим корпусом электромагнитное поле развивается независимо от внешней электромагнитной обстановки. Объемный заряд и радиационная проводимость в воздухе создаются электронами отдачи, эмитируемыми с внутренней поверхности корпуса.

Рассмотрим прямоугольную расчетную область в форме параллелепипеда со сторонами $L_{1}, L_{2}, L_{3}$ и идеально проводящей границей. Сплошная среда внутри расчетной области имеет проводимость $\sigma$ и диэлектрическую проницаемость $\varepsilon$. Электромагнитное поле в расчетной области создается сторонним током с плотностью $\mathbf{j}_{\text {ext }}=\mathbf{j}_{\text {ext }}(t, \mathbf{r})$, где $t$-лабораторное время, а $\mathbf{r}=(x, y, z)-$ декартовы координаты.

Внутри области электромагнитное поле описывается уравнениями Максвелла: 


$$
\operatorname{rot} \mathbf{H}=\frac{\varepsilon}{c} \frac{\partial \mathbf{E}}{\partial t}+\frac{4 \pi}{c}\left(\mathbf{j}_{\text {ext }}+\sigma \mathbf{E}\right), \quad \operatorname{rot} \mathbf{E}=-\frac{1}{c} \frac{\partial \mathbf{H}}{\partial t},
$$

где $\mathbf{E}=\mathbf{E}(t, \mathbf{r}), \mathbf{H}=\mathbf{H}(t, \mathbf{r})$ - векторы напряженности электрического и магнитного поля, $c$ - скорость света в вакууме.

Условия идеальной проводимости границы имеют вид равенства нулю тангенциальной компоненты напряженности электрического поля:

$$
\begin{aligned}
& \left.E_{y}\right|_{x=0, L_{1}}=\left.E_{z}\right|_{x=0, L_{1}}=0,\left.\quad E_{x}\right|_{z=0, L_{3}}=\left.E_{y}\right|_{z=0, L_{3}}=0, \\
& \left.E_{x}\right|_{y=0, L_{2}}=\left.E_{z}\right|_{y=0, L_{2}}=0 .
\end{aligned}
$$

Начальные условия считаются однородными: $\left.\mathbf{E}\right|_{t=0}=0,\left.\mathbf{H}\right|_{t=0}=0$.

Представим сторонний ток $\mathbf{j}_{\text {ext }}$ в виде произведения функций времени и пространственных переменных

$$
\mathbf{j}_{\text {ext }}=g(t) \mathbf{j}(x, y, z),
$$

где функция $g(t)$ отлична от 0 на интервале $\left(0,2 T_{0}\right), T_{0}$ - характерное время изменения стороннего тока. Будем считать, что функция $g(t)$ нормирована на единицу, то есть $\int_{0}^{2 T_{0}} g(t) d t=1$. Отметим для дальнейшего, что $g(t)$ и ее производные $g^{\prime}(t), g^{\prime \prime}(t), \ldots$ имеют величину порядка $g(t) \sim 1 / T_{0} \sim \omega_{0}$, $g^{\prime}(t) \sim 1 / T_{0}^{2} \sim \omega_{0}^{2}, g^{\prime \prime}(t) \sim 1 / T_{0}^{3} \sim \omega_{0}^{3}$, где $\omega_{0}=2 \pi / T_{0}$. Также положим, что вектор $\mathbf{j}$ имеет одну нетривиальную компоненту $\mathbf{j}=(0,0, j)$.

Следует отметить, что механизмы и скорость термализации электронов, образующихся при ударной ионизации типовых диэлектриков [16] и воздушной среды [15], существенно различны. Радиационная проводимость воздуха при нормальном давлении существенно превосходит радиационную проводимость диэлектрика при равных потоках тормозного излучения. Поэтому соотношения плотностей тока смещения и проводимости в уравнениях (1) существенно различны. В вакууме и в компаунде плотность тока проводимости мала по сравнению с плотностью тока смещения. Напротив, в воздушной среде с нормальным давлением развивается такая радиационная проводимость, что создаваемый ею ток существенно превосходит ток смещения. Рассмотрим оценки решения задачи (1-3) в указанных случаях.

\section{3. Уравнения Максвелла в проводящей среде}

Слагаемое $\frac{\varepsilon}{c} \frac{\partial E}{\partial t}$ в уравнении (1) имеет порядок величины $\frac{\varepsilon \omega_{0} E}{c}$. Рассмотрим ситуацию, при которой $\varepsilon \omega_{0} E \ll 4 \pi \sigma E$. 
Тогда плотностью тока смещения в (1) можно пренебречь:

$$
\operatorname{rot} \mathbf{H}=\frac{4 \pi}{c}(g(t) \mathbf{j}+\sigma \mathbf{E}), \quad \operatorname{rot} \mathbf{E}=-\frac{1}{c} \frac{\partial \mathbf{H}}{\partial t} .
$$

Будем искать решение (4) в виде

$$
\mathbf{E}=-\frac{4 \pi \sigma}{c^{2}} \frac{\partial \boldsymbol{\Pi}}{\partial t}+\operatorname{grad} \operatorname{div} \boldsymbol{\Pi}, \quad \mathbf{H}=\frac{4 \pi \sigma}{c} \operatorname{rot} \boldsymbol{\Pi},
$$

где $\Pi=(0,0, u(t, \mathbf{r}))-$ вектор Герца [17]. Выражения (5) для электрического и магнитного полей удовлетворяют уравнениям Максвелла, если $u$ удовлетворяет уравнению

$$
\Delta u-\frac{4 \pi \sigma}{c^{2}} \frac{\partial u}{\partial t}=-\frac{g j}{\sigma} .
$$

Дополним уравнение (6) начальными и граничными условиями

$$
\left.u\right|_{t=0}=0,\left.\quad \dot{u}\right|_{t=0}=0,\left.\quad u\right|_{x=0, L_{1}}=0,\left.\quad u\right|_{y=0, L_{2}}=0, \quad \partial u /\left.\partial z\right|_{z=0, L_{3}}=0
$$

и рассмотрим вспомогательную начально-краевую задачу (6), (7).

Компоненты электромагнитного поля выражаются через вектор Герца следующим образом:

$$
\begin{array}{ll}
E_{x}=\frac{\partial^{2} u}{\partial x \partial z}, & E_{y}=\frac{\partial^{2} u}{\partial y \partial z}, \quad E_{z}=\frac{\partial^{2} u}{\partial z^{2}}-\frac{4 \pi \sigma}{c^{2}} \frac{\partial u}{\partial t}, \\
H_{x}=\frac{4 \pi \sigma}{c} \frac{\partial u}{\partial y}, & H_{y}=-\frac{4 \pi \sigma}{c} \frac{\partial u}{\partial x}, \quad H_{z}=0 .
\end{array}
$$

Следствием выражений (8) и начальных условий для функции $u$ является выполнение начальных условий $\left.\mathbf{E}\right|_{t=0}=0,\left.\mathbf{H}\right|_{t=0}=0$.

Следствием выражений $E_{x}=\frac{\partial^{2} u}{\partial x \partial z}, E_{y}=\frac{\partial^{2} u}{\partial y \partial z}$ и граничного условия $\partial u /\left.\partial z\right|_{z=0, L_{3}}=0$ является выполнение условий $\left.E_{x}\right|_{z=0, L_{3}}=0,\left.E_{y}\right|_{z=0, L_{3}}=0$.

Следствием выражений $E_{x}=\frac{\partial^{2} u}{\partial x \partial z}, E_{y}=\frac{\partial^{2} u}{\partial y \partial z}$ и граничных условий $\left.u\right|_{x=0, L_{1}}=0$ и $\left.u\right|_{y=0, L_{2}}=0$ являются условия $\left.E_{x}\right|_{y=0, L_{2}}=0,\left.E_{y}\right|_{x=0, L_{1}}=0$.

Следствием выражения $E_{z}=\frac{\partial^{2} u}{\partial z^{2}}-\frac{4 \pi \sigma}{c^{2}} \frac{\partial u}{\partial t} \quad$ и граничных условий 
$\left.u\right|_{x=0, L_{1}}=0,\left.u\right|_{y=0, L_{2}}=0$ являются равенства

$$
\left.E_{z}\right|_{x=0, L_{1}}=-\left.\frac{4 \pi \sigma}{c^{2}} \frac{\partial u}{\partial t}\right|_{x=0, L_{1}},\left.\quad E_{z}\right|_{y=0, L_{2}}=-\left.\frac{4 \pi \sigma}{c^{2}} \frac{\partial u}{\partial t}\right|_{y=0, L_{2}} .
$$

Покажем, что решение вспомогательной задачи удовлетворяет условиям:

$$
\left.\frac{\partial u}{\partial t}\right|_{x=0, L_{1}}=\left.\frac{\partial u}{\partial t}\right|_{y=0, L_{2}}=0
$$

Электромагнитное поле (5) удовлетворяет уравнениям Максвелла (4) с однородными начальными данными и граничными условиями (2).

Рассмотрим решение вспомогательной задачи методом разложения по собственным функциям соответствующей задачи Штурма-Лиувилля [17]:

$$
\begin{aligned}
& u=\sum_{m, n, l} u_{m n l}(t) U_{m n l}(x, y, z), \quad U_{m n l}=\sin \left(\frac{\pi m x}{L_{1}}\right) \sin \left(\frac{\pi n y}{L_{2}}\right) \cos \left(\frac{\pi l z}{L_{3}}\right), \\
& u_{m n l}(t)=\frac{c^{2} j_{m n l}}{4 \pi \sigma^{2}} \int_{0}^{t} \exp \left\{-\Omega_{1} R_{m n l}^{2} \tau\right\} g(t-\tau) d \tau,
\end{aligned}
$$

где $\Omega_{1}=\frac{\omega_{1}^{2}}{4 \pi \sigma}, \omega_{1}=\frac{\pi c}{L_{3}}, R_{m m l}^{2}=\left(\frac{L_{3}}{L_{1}}\right)^{2} m^{2}+\left(\frac{L_{3}}{L_{2}}\right)^{2} n^{2}+l^{2}>1, j_{m n l}$ - коэффициенты разложения функции $j(x, y, z)$ в ряд Фурье по функциям $U_{m n l}$.

Следствием (10) является выполнение условий (9). Действительно, в соответствии с (10):

$$
\partial u / \partial t_{\substack{x=0, L_{1} \\ y=0, L_{2}}}=\left.\sum_{m, n, l} \dot{u}_{m n l}(t) U_{m n l}(x, y, z)\right|_{\substack{x=0, L_{1} \\ y=0, L_{2}}}=0 .
$$

Получим из (10) выражения для компонент электромагнитного поля:

$$
\begin{aligned}
& E_{x}=-\frac{L_{3}}{L_{1}} \frac{\Omega_{1}}{\sigma} \sum_{m, n, l} j_{m n l} V_{m n l} I_{m n l}(t) m l, \quad V_{m n l}=\cos \left(\frac{\pi m x}{L_{1}}\right) \sin \left(\frac{\pi n y}{L_{2}}\right) \sin \left(\frac{\pi l z}{L_{3}}\right), \\
& E_{y}=-\frac{L_{2}}{L_{1}} \frac{\Omega_{1}}{\sigma} \sum_{m, n, l} j_{m n l} W_{m n l} I_{m n l}(t) n l, \quad W_{m n l}=\sin \left(\frac{\pi m x}{L_{1}}\right) \cos \left(\frac{\pi n y}{L_{2}}\right) \sin \left(\frac{\pi l z}{L_{3}}\right), \\
& E_{z}=\frac{1}{\sigma} \sum_{m, n, l} j_{m n l} U_{m n l}\left[\Omega_{1}\left(R_{m n l}^{2}-l^{2}\right) I_{m n l}(t)-g(t)\right]
\end{aligned}
$$




$$
\begin{array}{ll}
H_{x}=\frac{L_{3}}{L_{2}} \frac{\omega_{1}}{\sigma} \sum_{m, n, l} j_{m n l} P_{m n l} I_{m n l}(t) n, \quad P_{m n l}=\sin \left(\frac{\pi m x}{L_{1}}\right) \cos \left(\frac{\pi n y}{L_{2}}\right) \cos \left(\frac{\pi l z}{L_{3}}\right), \\
H_{y}=\frac{L_{3}}{L_{1}} \frac{\omega_{1}}{\sigma} \sum_{m, n, l} j_{m n l} Q_{m n l} I_{m n l}(t) m, & Q_{m n l}=\cos \left(\frac{\pi m x}{L_{1}}\right) \sin \left(\frac{\pi n y}{L_{2}}\right) \cos \left(\frac{\pi l z}{L_{3}}\right) .
\end{array}
$$

Входящие в выражения (11) интегралы

$$
I_{m n l}(t)=\int_{0}^{t} \exp \left\{-\Omega_{1} R_{m n l}^{2}(t-\tau)\right\} g(\tau) d \tau
$$

можно представить в виде ряда по степеням $1 / \Omega_{1}$, применяя последовательное интегрирование по частям:

$$
\begin{aligned}
I_{m n l}(t) & =\sum_{k=0}^{N-1}\left[\left(g^{(k)}(t)-\exp \left\{-\Omega_{1} R_{m n l}^{2} t\right\} g^{(k)}(0)\right) / \Omega_{1}^{k+1} R_{m n l}^{2(k+1)}+\right. \\
& \left.+\int_{0}^{t} \exp \left\{-\Omega_{1} R_{m n l}^{2}(t-\tau)\right\} g^{(N)}(t) d \tau\right]
\end{aligned}
$$

Остаточный член ряда имеет порядок $\sigma^{N+1} \omega_{0}^{N+1} / \omega_{1}^{2(N+1)}$. Подставляя данное представление $I_{m n l}(t)$ без остаточного члена при $N=1$ в выражения для полей (11), получим

$$
\begin{aligned}
& E_{x}=-\frac{L_{3}}{L_{1}} \sum_{m, n, l} \frac{j_{m n l}}{\sigma} V_{m n l} \frac{m l}{R_{m n l}^{2}}\left[g(t)-\frac{g^{\prime}(t)-\exp \left\{-\Omega_{1} R_{m n l}^{2} t\right\} g^{\prime}(0)}{\Omega_{1} R_{m n l}^{2}}\right], \\
& E_{y}=-\frac{L_{2}}{L_{1}} \sum_{m, n, l} \frac{j_{m n l}}{\sigma} W_{m n l} \frac{n l}{R_{m n l}^{2}}\left[g(t)-\frac{g^{\prime}(t)-\exp \left\{-\Omega_{1} R_{m n l}^{2} t\right\} g^{\prime}(0)}{\Omega_{1} R_{m n l}^{2}}\right], \quad(12) \\
& E_{z}=-\sum_{m, n, l} \frac{j_{m n l}}{\sigma} U_{m n l} \times\left[\frac{l^{2}}{R_{m n l}^{2}} g(t)+\frac{R_{m n l}^{2}-l^{2}}{R_{m n l}^{4}} \frac{g^{\prime}(t)-\exp \left\{-\Omega_{1} R_{m n l}^{2} t\right\} g^{\prime}(0)}{\Omega_{1}}\right], \\
& H_{x}=\frac{L_{3}}{L_{2}} \frac{4 \pi}{\omega_{1}} \sum_{m, n, l} j_{m n l} P_{m n l}(x, y, z) \frac{n}{R_{m n l}^{2}}\left[g(t)-\frac{g^{\prime}(t)-\exp \left\{-\Omega_{1} R_{m n l}^{2} l\right\} g^{\prime}(0)}{\Omega_{1} R_{m n l}^{2}}\right], \\
& H_{y}=-\frac{L_{3}}{L_{1}} \frac{4 \pi}{\omega_{1}} \sum_{m, n, l} j_{m n l} Q_{m n l} \frac{m}{R_{m n l}^{2}}\left[g(t)-\frac{g^{\prime}(t)-\exp \left\{-\Omega_{1} R_{m n l}^{2} t\right\} g^{\prime}(0)}{\Omega_{1} R_{m n l}^{2}}\right] .
\end{aligned}
$$

Вторые слагаемые в (12) имеют порядок величины $\omega_{0} \sigma / \omega_{1}^{2}$. Если 
функция $g(t)$ такова, что $\omega_{0} \ll \omega_{1}^{2} / \sigma$, ими можно пренебречь. В этом случае электромагнитное поле с достаточной точностью зависит только от значений стороннего тока в текущий момент времени.

Это означает, что при вычислениях поля в момент времени $t^{\prime}$ функцию $g(t)$ можно заменить на некоторую другую функцию $f(t)$, с которой время счета будет существенно меньше. Действительно, пусть характерная частота $\omega_{0}$ функции $g(t)$ удовлетворяет условию $\omega_{0} \ll \omega_{1}^{2} / \sigma$. Подберем функцию $f(t)$ так, чтобы ее характерная частота $v_{0}$ удовлетворяла неравенствам $\omega_{0} \ll v_{0} \ll \omega_{1}^{2} / \sigma$, а в момент времени $t^{\prime \prime}$ выполнялось условие $f\left(t^{\prime \prime}\right)=g\left(t^{\prime}\right)$. В обоих случаях значения электромагнитного поля с хорошей точностью будут определяться величинами $f\left(t^{\prime \prime}\right)=g\left(t^{\prime}\right)$ и поэтому будут одинаковыми. В силу условия $v_{0} \gg \omega_{0}$ значение $t^{\prime \prime}$ можно выбрать много меньшим $t^{\prime}$, что пропорционально уменьшит время расчета.

Отметим особенность задачи моделирования электромагнитного поля фотонами. Поле создается электронами отдачи. Интенсивность их генерации при рассеянии импульса фотонов тормозного излучения равна нулю в нулевой момент времени. Поэтому плотность тока в нулевой момент времени равна нулю вместе со своей первой производной:

$$
\left.\mathbf{j}_{\text {ext }}\right|_{t=0}=\left.\frac{\partial \mathbf{j}_{\text {ext }}}{\partial t}\right|_{t=0}=0 .
$$

Другими словами, функция распределения электронов в фазовом пространстве подчиняется кинетическому уравнению с интенсивностью их генерации в правой части. В нулевой момент времени правая часть равна нулю. Отсюда следует, что $g^{\prime}(0)$, что существенно упрощает формулы (12).

\section{4. Уравнения Максвелла в диэлектрической среде}

Рассмотрим задачу (1-2) с однородными начальными условиями в приближении малой проводимости среды в расчетной области. Сторонний ток $\mathbf{j}_{e x t}$ представим в виде произведения аналогично (3).

Следствием однородных начальных условий, самих уравнений и соотношения (13) является условие $\left.\dot{\mathbf{E}}\right|_{t=0}=0,\left.\dot{\mathbf{H}}\right|_{t=0}=0$.

Будем предполагать, что для любой частоты электрического поля $\omega$ выполняется следующее условие $\varepsilon \omega E(\omega) \gg 4 \pi \sigma E(\omega)$, где $E(\omega)$ - частотный образ напряженности электрического поля. Тогда уравнения (1) приобретают вид

$$
\operatorname{rot} \mathbf{H}=\frac{\varepsilon}{c} \frac{\partial \mathbf{E}}{\partial t}+\frac{4 \pi}{c} g \mathbf{j}, \quad \operatorname{rot} \mathbf{E}=-\frac{1}{c} \frac{\partial \mathbf{H}}{\partial t}
$$


с начальными условиями $\left.\mathbf{E}\right|_{t=0}=0,\left.\mathbf{H}\right|_{t=0}=0,\left.\dot{\mathbf{E}}\right|_{t=0}=0,\left.\dot{\mathbf{H}}\right|_{t=0}=0$ и граничными условиями (2). Будем искать решение в виде

$$
\mathbf{E}=\operatorname{grad} \operatorname{div} \boldsymbol{\Pi}-\frac{\varepsilon}{c^{2}} \frac{\partial^{2} \boldsymbol{\Pi}}{\partial t^{2}}, \quad \mathbf{H}=\frac{\varepsilon}{c} \operatorname{rot} \frac{\partial \boldsymbol{\Pi}}{\partial t},
$$

где $\boldsymbol{\Pi}=(0,0, u(t, \mathbf{r}))-$ вектор Герца.

Электрическое и магнитное поля, представленные в таком виде, удовлетворяют уравнениям Максвелла, если $u$ удовлетворяет уравнению

$$
\frac{\partial}{\partial t} \Delta u-\frac{\varepsilon}{c^{2}} \frac{\partial^{3} u}{\partial t^{3}}=-\frac{4 \pi j}{\varepsilon} g(t) .
$$

Потребуем выполнения следующих начальных условий для функции $u$ :

$$
\left.u\right|_{t=0}=\left.\ddot{u}\right|_{t=0}=\left.\dddot{u}\right|_{t=0}=0 \text {. }
$$

Интегрируя уравнение (14) с учетом (15) от 0 до $t$, получим:

$$
\Delta u-\frac{\varepsilon}{c^{2}} \frac{\partial^{2} u}{\partial t^{2}}=-\frac{4 \pi j}{\varepsilon} G(t),
$$

где $G(t)=\int_{0}^{t} g(t) d t$.

Рассмотрим вспомогательную начально-краевую задачу для уравнения (16) с начальными условиями (15) и граничными условиями (7).

Компоненты электромагнитного поля выражаются через вектор Герца следующим образом:

$$
\begin{aligned}
& E_{x}=\frac{\partial^{2} u}{\partial x \partial z}, \quad E_{z}=\frac{\partial^{2} u}{\partial z^{2}}-\frac{\varepsilon}{c^{2}} \frac{\partial^{2} u}{\partial t^{2}}, \quad E_{y}=\frac{\partial^{2} u}{\partial y \partial z}, \\
& H_{x}=\frac{\varepsilon}{c} \frac{\partial^{2} u}{\partial t \partial y}, \quad H_{y}=-\frac{\varepsilon}{c} \frac{\partial^{2} u}{\partial t \partial x}, \quad H_{z}=0 .
\end{aligned}
$$

Следствием выражений (17) и начальных условий для функции $u$ является выполнение начальных условий $\left.\mathbf{E}\right|_{t=0}=0,\left.\mathbf{H}\right|_{t=0}=0,\left.\dot{\mathbf{E}}\right|_{t=0}=0,\left.\dot{\mathbf{H}}\right|_{t=0}=0$.

Граничные условия для функции $u$ обеспечивают выполнение граничных условий для напряженности электромагнитного поля аналогично предыдущему разделу.

Решая вспомогательную задачу методом разложения по собственным функциям соответствующей задачи Штурма-Лиувилля, получим: 


$$
u=\sum_{m, n, l} u_{m n l}(t) U_{m n l}(x, y, z), \quad U_{m n l}=\sin \left(\frac{\pi m x}{L_{1}}\right) \sin \left(\frac{\pi n y}{L_{2}}\right) \cos \left(\frac{\pi l z}{L_{3}}\right),
$$

где коэффициенты $u_{m n l}(t)$ удовлетворяют следующему уравнению и начальным условиям:

$$
\begin{aligned}
& \ddot{u}_{m n l}+\Omega_{m n l}^{2} u_{m n l}=\frac{4 \pi c^{2}}{\varepsilon^{2}} j_{m n l} G(t), \quad \Omega_{m n l}=\frac{\omega_{1} r_{m n l}}{\sqrt{\varepsilon}}, \\
& \left.u_{m n l}\right|_{t=0}=\left.\ddot{u}_{m n l}\right|_{t=0}=\left.\dddot{u}_{m n l}\right|_{t=0}=0 .
\end{aligned}
$$

Решением уравнения (18) является функция

$$
u_{m n l}(t)=\frac{4 \pi c^{2}}{\varepsilon^{2}} \frac{j_{m n l}}{\Omega_{m n l}} \int_{0}^{t} \sin \Omega_{m n l}(t-\tau) G(\tau) d \tau .
$$

Получим выражения для компонент электромагнитного поля:

$$
\begin{aligned}
& E_{z}=-\frac{4 \pi}{\varepsilon} \sum_{m, n, l} j_{m n l} U_{m n l}(x, y, z)\left[\frac{l^{2}}{r_{m n l}^{2}} G(t)+\frac{r_{m n l}^{2}-l^{2}}{r_{m n l}^{2}} I_{m n l}(t)\right], \\
& E_{x}=-\frac{4 \pi}{\varepsilon} \frac{L_{3}}{L_{1}} \sum_{m, n, l} j_{m n l} V_{m n l}(x, y, z) \frac{m l}{r_{m n l}^{2}}\left[G(t)-I_{m n l}(t)\right], \\
& E_{y}=-\frac{4 \pi}{\varepsilon} \frac{L_{3}}{L_{2}} \sum_{m, n, l} j_{m n l} W_{m n l}(x, y, z) \frac{n l}{r_{m n l}^{2}}\left[G(t)-I_{m n l}(t)\right], \\
& H_{x}=\frac{4 \pi}{\sqrt{\varepsilon}} \frac{L_{3}}{L_{2}} \sum_{m, n, l} j_{m n l} P_{m n l}(x, y, z) \frac{n}{r_{m n l}} J_{m n l}(t), \\
& H_{y}=-\frac{4 \pi}{\sqrt{\varepsilon}} \frac{L_{3}}{L_{1}} \sum_{m, n, l} j_{m n l} Q_{m n l}(x, y, z) \frac{m}{r_{m n l}} J_{m n l}(t),
\end{aligned}
$$

где $I_{m n l}(t)=\int_{0}^{t} \cos \Omega_{m n l}(t-\tau) g(\tau) d \tau, J_{m n l}(t)=\int_{0}^{t} \sin \Omega_{m n l}(t-\tau) g(\tau) d \tau$.

Выражения (19) содержат интегралы $I_{m n l}(t)$ и $J_{m n l}(t)$ от произведения быстро осциллирующих функций на медленно меняющуюся функцию $g(t)$. Очевидно, что они малы по сравнению с $G(t)$ и в первом приближении ими можно пренебречь. Чтобы получить дальнейшие приближения, запишем $I_{m n l}(t)$ и $J_{m n l}(t)$ в виде:

$$
I_{m n l}(t)=\cos \Omega_{m n l} t \int_{0}^{t} d \tau g(\tau) \cos \Omega_{m n l} \tau+\sin \Omega_{m n l} t \int_{0}^{t} d \tau g(\tau) \sin \Omega_{m n l} \tau,
$$




$$
J_{m n l}(t)=\sin \Omega_{m n l} t \int_{0}^{t} d \tau g(\tau) \cos \Omega_{m n l} \tau-\cos \Omega_{m n l} t \int_{0}^{t} d \tau g(\tau) \sin \Omega_{m n l} \tau .
$$

Интегралы можно вычислить последовательным интегрированием по частям [19]. В результате получим ряд по степеням $1 / \Omega_{m n l}$ :

$$
\begin{aligned}
& I_{m n l}(t)=\sum_{k=0}^{K-1} \frac{g^{(k)}(0)}{\Omega_{m n l}^{k+1}}\left\{\begin{array}{l}
(-1)^{k / 2} \sin \Omega_{m n l} t, k=2 n \\
(-1)^{(k+1) / 2} \cos \Omega_{m n l} t, k=2 n+1
\end{array}\right\}- \\
& -\sum_{k=0}^{K-1} \frac{g^{(k)}(t)}{\Omega_{m n l}^{k+1}}\left\{\begin{array}{l}
0, k=2 n \\
(-1)^{(k+1) / 2}, k=2 n+1
\end{array}\right\}+o\left(\frac{\omega_{0}^{K}}{\Omega_{m n l}^{K}}\right), \\
& J_{m n l}(t)=\sum_{k=0}^{K-1} \frac{g^{(k)}(0)}{\Omega_{m n l}^{k+1}}\left\{\begin{array}{l}
(-1)^{(k+2) / 2} \cos \Omega_{m n l} t, k=2 n \\
(-1)^{(k+1) / 2} \sin \Omega_{m n l} t, k=2 n+1
\end{array}\right\}+ \\
& +\sum_{k=0}^{K-1} \frac{g^{(k)}(t)}{\Omega_{m n l}^{k+1}}\left\{\begin{array}{l}
(-1)^{k / 2}, k=2 n \\
0, k=2 n+1
\end{array}\right\}+o\left(\frac{\omega_{0}^{K}}{\Omega_{m n l}^{K}}\right) .
\end{aligned}
$$

В частности, при $K=2$ с учетом (13):

$$
I_{m n l}(t)=\frac{g^{\prime}(t)}{\Omega_{m n l}^{2}}+o\left(\frac{\omega_{0}^{2}}{\Omega_{m n l}^{2}}\right), \quad J_{m n l}(t)=\frac{g(t)}{\Omega_{m n l}}+o\left(\frac{\omega_{0}^{2}}{\Omega_{m n l}^{2}}\right) .
$$

Тогда с точностью до слагаемых порядка $\omega_{0}^{2} / \Omega^{2}$.

$$
\begin{aligned}
& E_{z}=-\frac{4 \pi}{\varepsilon} \sum_{m, n, l} j_{m n l} U_{m n l}(x, y, z)\left[\frac{l^{2}}{r_{m n l}^{2}} G(t)+\frac{r_{m n l}^{2}-l^{2}}{r_{m n l}^{4}} \frac{\varepsilon g^{\prime}(t)}{\omega_{1}^{2}}\right], \\
& E_{y}=-\frac{4 \pi}{\varepsilon} \frac{L_{2}}{L_{1}} \sum_{m, n, l} j_{m n l} W_{m n l}(x, y, z) \frac{n l}{r_{m n l}^{2}}\left[G(t)-\frac{\varepsilon g^{\prime}(t)}{r_{m n l}^{2} \omega_{1}^{2}}\right], \\
& E_{x}=-\frac{4 \pi}{\varepsilon} \frac{L_{3}}{L_{1}} \sum_{m, n, l} j_{m n l} V_{m n l}(x, y, z) \frac{m l}{r_{m n l}^{2}}\left[G(t)-\frac{\varepsilon g^{\prime}(t)}{r_{m n l}^{2} \omega_{1}^{2}}\right], \\
& H_{x}=\frac{4 \pi}{\omega_{1}} \frac{L_{3}}{L_{2}} \sum_{m, n, l} j_{m n l} P_{m n l}(x, y, z) \frac{n}{r_{m n l}^{2}} g(t), \\
& H_{y}=-\frac{4 \pi}{\omega_{1}} \frac{L_{3}}{L_{1}} \sum_{m, n, l} j_{m n l} Q_{m n l}(x, y, z) \frac{m}{r_{m n l}^{2}} g(t) .
\end{aligned}
$$


Выражения (20) показывают, что электромагнитное поле с хорошей точностью описывается первыми слагаемыми, пока $\omega_{1} / \omega_{0} \gg \sqrt{\varepsilon}$.

В этом приближении электрическое поле не зависит от скорости света, а магнитное поле обратно пропорционально ему. Значит, при численном расчете на основе алгоритма для полной системы уравнений Максвелла скорость света можно уменьшить. Это не изменит результатов расчета электрического поля. Значения магнитного поля следует уменьшить во столько же ра3, во сколько уменьшена скорость света. Уменьшение скорости света позволит пропорционально увеличить шаг по времени. Очевидно, что уменьшению скорости света не следует придавать физического смысла.

\section{5. Заключение}

Низкочастотное электромагнитное поле радиационного происхождения в областях с идеально проводящей границей возможно моделировать с помощью численного алгоритма, основанного на явной разностной схеме для уравнений Максвелла в полной постановке.

Для этого необходимо рассмотреть уравнения Максвелла (1) в произвольной расчетной области с идеально проводящей границей и сделать следующие предположения.

Пусть правая часть уравнений - плотность стороннего тока - представима в виде произведения (3), причем все компоненты вектора $\mathbf{j}$ могут быть отличными от нуля. Функцию $g(t)$ будем считать достаточно гладкой.

Также предположим, что в области развивается радиационная проводимость, достаточная для того, чтобы плотность тока проводимости существенно превысила плотность тока смещения.

Тогда низкочастотное электромагнитное поле вычисляется следующим образом.

На интервале $\left(0,2 T_{0}\right)$ выделяются моменты времени $t=t_{i}$, для которых актуально вычисление электромагнитного поля. Для каждого $t_{i}$ строится функция $f_{i}(t)$, удовлетворяющая условиям:

$$
\operatorname{supp}\left(f_{i}\right)=\left(t_{i}-\Delta t_{i}, 2 T_{0}\right), \quad \Delta t_{i} \sim 1 / \sigma\left(t_{i}\right),\left.\quad f_{i}\right|_{t_{i}-\Delta t}=\left.\frac{\partial f_{i}}{\partial t}\right|_{t_{i}-\Delta t}=0,
$$

$f_{i}(t)<1$ при $t_{i}-\Delta t_{i}<t<t_{i}, f_{i}(t)=1$ при $t \geq t_{i}$.

Электромагнитное поле вычисляется по явной разностной схеме для уравнений Максвелла в полной постановке с правой частью $\mathbf{j}_{\text {ext }}^{i}=f_{i}(t) \mathbf{j}_{\text {ext }}$. Расчет ведется с момента времени $t=t_{i}-\Delta t_{i}$ с начальными условиями $\left.\mathbf{E}\right|_{t=t_{i}-\Delta t_{i}}=\left.\mathbf{H}\right|_{t=t_{i}-\Delta t_{i}}=0$. Для $t \geq t_{i}$ расчет ведется до установления решения с 
учетом частот, соответствующих собственными модам расчетной области.

В результате решение вычисляется для некоторого характерного набора моментов времени $t=t_{i}$, определяемого свойствами функции $g(t)$.

В противном случае, если радиационная проводимость в расчетной области существенно меньше тока смещения, то расчеты проводятся с уменьшенной скоростью света. Условие $\omega_{1} / \omega_{0} \gg \sqrt{\varepsilon}$, означает, что

$$
c \gg 2 L \sqrt{\varepsilon} / T_{0},
$$

где $L$ - характерный размер области, ограниченной идеально проводящей границей.

Пусть область с характерным размером $L=10$ см заполнена эпоксидным компаундом с диэлектрической проницаемостью 4 и облучается тормозным излучением в течение времени $T_{0}=1 \mathrm{c}$. Тогда условие (22) приобретает вид $c \gg 40 \mathrm{~cm} / \mathrm{c}$. Выбирая $c=10^{4} \mathrm{~cm} / \mathrm{c}$, получаем возможность увеличения временного шага на 6 порядков в численном алгоритме, основанном на явной разностной схеме для уравнений Максвелла в полной постановке.

Уменьшение скорости света исключает из спектра решения частоты, соответствующие собственным модам расчетной области. Счет на установление в проводящей среде сохраняет решение исходной задачи только если моменты времени $t_{i}$ выбраны так, что $\omega_{1}\left(t_{i}-t_{i-1}\right) \ll 1$. В обоих случаях собственные моды теряются.

Несмотря на потерю собственных мод, предложенные подходы к моделированию позволяют с достаточной точностью моделировать электромагнитное поле, создаваемое радиационными воздействиями большой длительности в экранированных блоках радиоэлектронной аппаратуры. При этом используется модель, основанная на уравнениях Максвелла в полной постановке, без существенной доработки программного обеспечения.

\section{СПИСОК ЛИТЕРАТУРЫ}

1. G. Watkin's. Radiation Damage in Semiconductors. - N.Y.: Academic Press, 1965.

2. L.D. Landau, E.M. Lifshitz, L.P. Pitaevskii. Electrodynamics of Continuous Media, 1st Edition, Butterworth-Heinemann, 1984, v.8.

3. A.V. Berezin, A.A. Kriukov, B.D. Pliushchenkov. The method of electromagnetic field with the given wavefront calculation» // MM\&CS, 2011, v.23 (3), p.109-126.

4. B. Bakholdin, A.V. Berezin, A.A. Kryukov, M.B. Markov, B.D. Plyushchenkov, D.N. Sadovnichii. Electromagnetic wave in the medium with dispersion of dielectric permittivity // Math. Models \& Comp. Simul., 2017, v.9, №2, p.190-200.

5. A.V. Berezin, A.S. Vorontsov, S.V. Zakharov, M.B. Markov, S.V. Parot'kin. Modeling of gaseous discharge's electron stage // MM\&CS, 2013, v.5, №5, p.492-500. 
6. S.K. Godunov, V.S. Riabenkiy. Difference Schemes: An Introduction to the Underlying Theory. Transl. from the Russian by E.M. Gelbard. Studies in Mathematics and its Applications. V.19. Amsterdam; New York; North-Holland, Wiley, 1964, 289 p.

7. Boris J.P., Book D.L. Flux-corrected transport // J. Comp. Phys., 1973, v.11, №1, p.38-69; 1975, v.18, №3, p.248-283

8. S.N. Vernov, A.E. Chudakov. Terrestrial corpuscular and cosmic rays // Space Research. H. Kallmann Bijl (eds). - Amsterdam: 1960, p.751-796.

9. H. Davies, H.A. Bethe and L.C. Maximon. Theory of bremsstrahlung and pair production. Integral cross section for pair production // Phys. Rev., 1954, v.93, p.788-795.

10. L.D. Landau, E.M. Lifshitz. The Classical Theory of Fields, 4th Edition, ButterworthHeinemann, 1975, v.2.

11. W. Heitler. The Quantum Theory of Radiation. - Oxford: Clarendon Press, 1954.

12. N.F. Mott, H.S.W. Massey. The theory of atomic collisions. - Oxford: Clarendon Press, 1965.

13. M. Gryzinski. Classic Theory of Electronic and Ionic Inelastic Collisions // Phys. Rev., 1959, v.115, №2, p.374-383.

14. Yong-Ki Kim, M.E. Rudd. Theory for Ionization of Molecules by Electrons // Phys. Rev., 1994, v.50, p.3954-3967.

15. M.B. Markov, S.V. Parot'kin. The kinetic model of radiation-induced gas conductivity // Mathematical Models and Computer Simulations, 2011, v.3, №6, p.712-722.

16. Д.Н. Садовничий, А.П. Тютнев, С.А. Хатипов, Ю.А. Милиџин. Радиационная электропроводность резин и метод ее прогнозирования // Химия высоких энергий, 1998, т.32, №1, с.7-13;

D.N. Sadovnichij, A.P. Tiutnev, S.A. Khatipov, Iu.A. Militsin. Radiatsionnaya elektroprovodnost rezin i metod ee prognozirovaniyia // Khimiia vysokikh energij, 1998, t.32, №1, s.7-13.

17. А.С. Ильинский, В.В. Кравцов, А.Г. Свешников. Математические модели электродинамики. - М.: Высшая школа, 1991;

A.S. Ilyinskiy, V.V. Kravtsov, A.G. Sveshnikov. Matematicheskie modeli elektrodinamiki.M.: Vysshaya shkola, 1991.

18. А.Н. Тихонов, А.А. Самарский. Уравнения математической физики. - М.: Наука, 1977. A.N. Tikhonov, A.A. Samarskiy. Uravneniia matematicheskoy fiziki. - M.: Nauka, 1977.

19. A. Erdélyi. Asymptotic expansions // Dover Publications, 1956.

Поступила в редакцию 17.04.2020

После доработки 17.04.2020

Принята к публикации 18.05.2020 